\title{
Allelopathic Prenylflavanones from the Fallen Leaves of Macaranga tanarius
}

\author{
Mei-Huims Tseng, ${ }^{\dagger}$ Chang-Hung Chou, ${ }^{\dagger}$ Yih-Ming Chen $^{\dagger}{ }^{\dagger}$ and Yueh-Hsiung Kuo*,\$
}

Departments of Botany and Chemistry, National Taiwan University, Taipe, Taiwan 106, Institute of Botany, Academia Sinica, Taipe, Taiwan 115, and Department of Biological Sciences, National Sun Yat-sen University, Kaoshing 804, Taiwan, Republic of China

Received J anuary 26, 2001

Two new prenylflavanones, tanariflavanones A (1), and B (2), and one known compound, (-)-nymphaeol-C (3), were isolated from the fallen leaves of Macaranga tanarius. Compounds $\mathbf{1}$ and $\mathbf{2}$ showed inhibition of radicle growth of lettuce seedlings at $200 \mathrm{ppm}$. Their structures were elucidated primarily by NMR, circular dichroism, and mass spectroscopic methods.

The genus Macaranga is one of the largest genera of the Euphorbiaceae, with approximately 300 species. $^{1}$ Previously reported components from the species of this genus include diterpenoids, triterpenoids, steroids, ${ }^{2,3}$ and hydrolyzable tannins ${ }^{4}$ from $M$. tanarius, a prenyl stilbene, vedelianin, ${ }^{5}$ and a geranyl flavonol ${ }^{6}$ from M. vedeliana, antibacterial prenylated flavanones ${ }^{7}$ from M. plejostemona, chromenoflavones ${ }^{8}$ from $M$. indica, and cytotoxic geranyl stilbenes ${ }^{9}$ from M. schweinfurthi i. M. tanarius is a common tropical tree distributed from southern Asia to northern Australia. ${ }^{10}$ The allel opathic potential of the fallen leaves of $M$. tanarius was indicated from field observations and bioassays, prompting us to study its chemical components. A methanol extract of the fallen leaves was suspended in $\mathrm{H}_{2} \mathrm{O}$ and then partitioned with ethyl acetate. The ethyl acetate solubles were fractionated to yield two new prenylflavanones, tanariflavanones A (1) and B (2), and the known (-)-nymphaeol-C (3). ${ }^{11}$ This paper describes the structural elucidation of $\mathbf{1}$ and $\mathbf{2}$ and their inhibitory effect on radicle growth of germinating lettuce seeds.

The mol ecular formula $\left(\mathrm{C}_{30} \mathrm{H}_{36} \mathrm{O}_{7}\right)$ of $\mathbf{1}$, showing one more oxygen atom than 3, was obtained from its HREIMS and ${ }^{13} \mathrm{C}$ NMR data. The I R spectrum of $\mathbf{1}$ indicated the presence of hydroxyl $\left(3422 \mathrm{~cm}^{-1}\right)$, conjugated carbonyl $\left(1647 \mathrm{~cm}^{-1}\right)$, and aromatic ( 1610 and $1500 \mathrm{~cm}^{-1}$ ) groups. Its UV spectrum exhibited maxima at 231 (sh) and $293 \mathrm{~nm}$. The ${ }^{1} \mathrm{H}$ NMR spectrum of $\mathbf{1}$ exhibited signals for a phenolic $\mathrm{OH}$ at $\delta 12.35$ (s, OH-5), which was a hydrogen strongly bonded to the 4-carbonyl group. An ABX system at $\delta 5.48(1 \mathrm{H}, \mathrm{dd}$, $\mathrm{J}=12.8,2.8 \mathrm{~Hz}), 2.73(1 \mathrm{H}, \mathrm{dd}, \mathrm{J}=17.2,2.8 \mathrm{~Hz})$, and 3.12 $(1 \mathrm{H}, \mathrm{dd}, \mathrm{J}=17.2,12.8 \mathrm{~Hz}$ ) was diagnostic for $\mathrm{H}-2$ and $\mathrm{H}-3$ of a flavanone nucleus. ${ }^{11}$ The appearance of three ol efinic methyl groups $(\delta 1.57,1.64,1.74)$, four multiplet methylene protons $(\delta 2.06)$, two benzylic methylene protons $(\delta 3.42$, br $\mathrm{d}, \mathrm{J}=6.8 \mathrm{~Hz}$ ), and two triplet vinyl protons $(\delta 5.02$, 5.17) indicated the presence of a geranyl group. ${ }^{11}$ The presence of a 2,2-dimethylchromane moiety with a secondary hydroxy group was revealed by signals at $\delta 1.31$ and 1.35 ( $3 \mathrm{H}$ each, s), $2.64\left(1 \mathrm{H}, \mathrm{dd}, \mathrm{J}=16.8,5.2 \mathrm{~Hz}, \mathrm{H}_{\mathrm{a}}-\mathrm{I}^{\prime \prime \prime}\right)$, $2.87\left(1 \mathrm{H}, \mathrm{dd}, \mathrm{J}=16.8,5.2 \mathrm{~Hz}, \mathrm{H}_{\mathrm{b}}-\mathrm{I}^{\prime \prime \prime}\right)$, and $3.83(1 \mathrm{H}, \mathrm{t}, \mathrm{J}$ $\left.=5.2 \mathrm{~Hz}, \mathrm{H}-2^{\prime \prime \prime}\right)$. The geranyl group was placed at $\mathrm{C}-2^{\prime}$ due to $\mathrm{H}_{2}-\mathrm{I}^{\prime \prime}$ and $\mathrm{H}-2$ having NOESY correlation. There was also a significant MS fragment at $\mathrm{m} / \mathrm{z} 384\left[\mathrm{M}^{+}-124\right] .^{11}$ The absence of a bathochromic shift in the UV spectrum of $\mathbf{1}$, after the addition of aluminum chloride, indicated a substituent at C-6. ${ }^{7}$ The methylene protons at $\delta 2.64$ and

* To whom correspondence should be addressed. Tel: + 88623638146. Fax: + 886-2-23636359. E-mail: yhkuo@ccms.ntu.edu.tw.

† Department of Botany, National Taiwan University.

₹ Institute of Botany, Academia Sinica, and Department of Biological Sciences, National Sun Yat-sen University.

$\S$ Department of Chemistry, National Taiwan University.<smiles>CC(C)=CCCC(C)=C[CH]c1c([C]2CC(=O)c3c(cc4c(c3O)C(F)[C@H](O)C(F)(F)O4)O2)ccc(O)c1O</smiles><smiles>CC(C)=CCCC1(C)C=Cc2c([C@H]3CC(=O)c4c(ccc(O)c4O)O3)ccc(O)c2O1</smiles>

2<smiles>CC(C)=CCC/C(C)=C/Cc1c([C@H]2CC(=O)c3c(cc(O)c(CC=C(C)C)c3O)O2)ccc(O)c1O</smiles>

2.87 were assigned to a benzylic methylene. The coupling constant between $\mathrm{H}-\mathrm{I}^{\prime \prime \prime}$ and $\mathrm{H}-2^{\prime \prime \prime}$ clearly indicated that the hydroxyl group at C-2"' occupied an axial position. Therefore, 2-hydroxy-3,3-dimethylchromane must fuse on ring $\mathrm{A}$. The ortho-coupled signals at $\delta 6.80$ and $6.96 \mathrm{~J}=$ 8.4 Hz, $\left.\mathrm{H}-5^{\prime},-6^{\prime}\right)$ as well as $\mathrm{H}-6^{\prime}$ and $\mathrm{H}-3$ having NOESY correlation suggested that $\mathrm{C}-\mathrm{I}^{\prime},-2^{\prime},-3^{\prime}$, and $-4^{\prime}$ positions in ring $B$ were substituted. Therefore, two phenolic hydroxyl groups ( $\delta 5.56$ and 5.89 , exchangeable) were assigned to C-3' and C-4'. Thus, compound $\mathbf{1}$ was deduced to 
be 2'-geranyl- 5,3',4'-trihydroxy-6,7-(2,2-dimethyl-3-hydroxychromano)flavanone. The absol ute stereochemistry at C-2 of $\mathbf{1}$ was established as $\mathbf{S}$ based upon a positive extremum at $344 \mathrm{~nm}(\theta=+13590)$ and a negative extremum at 291 $\mathrm{nm}(\theta=-34010)$ in its circular dichroism spectrum. ${ }^{12}$

Compound 2 had the molecular formula $\mathrm{C}_{30} \mathrm{H}_{34} \mathrm{O}_{6}$ on the basis of HREIMS and ${ }^{13} \mathrm{C}$ NMR data. The IR spectrum of 2 showed bands attributed to hydroxyl $\left(3374 \mathrm{~cm}^{-1}\right)$, conjugated carbonyl (1641 cm-1), and aromatic (1606 and 1449 $\mathrm{cm}^{-1}$ ) groups. I ts UV spectrum exhibited maxima at 227 (sh), 274, and $285 \mathrm{~nm}$. Comparison of ${ }^{13} \mathrm{C}$ NMR data of 2 with those of known (-)-nymphaeol-C (3) suggested that 2 possessed the same skeletal structure as 3. Closer inspection of ${ }^{1} \mathrm{H},{ }^{13} \mathrm{C}$, and DEPT NMR spectra of $\mathbf{2}$ revealed the presence of three methyls $\left(\delta_{\mathrm{H}} 1.41,1.55\right.$, and 1.65; $\delta_{\mathrm{C}}$ 17.6, 25.6, and 26.1), two methylenes ( $\delta_{C} 40.7$ and 22.7), one oxygenated quaternary carbon $\left(\delta_{C} 79.0\right)$, and two double bonds ( $\delta_{C} 118.9$ and 130.9; 123.7 and 132.1). In the ${ }^{1} \mathrm{H}$ NMR spectrum of $\mathbf{2}$, vicinally coupled protons at $\delta 5.65$ and $6.58(\mathrm{~J}=10.0 \mathrm{~Hz})$ indicated that an oxygen atom at C-3' must have cyclized onto C-3" to form a pyran ring as shown in the structure 2 . This arrangement was supported by the observation of a fragment at $\mathrm{m} / \mathrm{z} 407\left[\mathrm{M}-\mathrm{CH}_{2-}\right.$ $\left.\mathrm{CH}_{2} \mathrm{CH}=\mathrm{C}(\mathrm{Me})_{2}\right]^{+}$in the EIMS of $2^{13}$ The ${ }^{1} \mathrm{H} \mathrm{NMR}$ spectrum $[\delta 1.74,1.80$ (each $3 \mathrm{H}, \mathrm{s}), 3.32(2 \mathrm{H}, \mathrm{br} \mathrm{d}, \mathrm{J}=5.8$ $\left.\mathrm{Hz}, \mathrm{H}-\mathrm{I}^{\prime \prime \prime}\right), 5.23\left(\mathrm{H}\right.$, br $\left.\left.\mathrm{t}, \mathrm{H}-2^{\prime \prime \prime}\right)\right]$ further showed the presence of one 1,1-dimethylallyl group. This group was placed at C-6, as $\mathbf{2}$ also showed no bathchromic shift on the addition of aluminum chloride. Compound $\mathbf{2}$ had the same absolute configuration (2 S) as $\mathbf{1}$ and $\mathbf{3}$ on the basis of the positive Cotton effect at $340 \mathrm{~nm}(\theta=+12390)$ and the negative Cotton effect at $287 \mathrm{~nm}(\theta=-16570)$. The structure of $\mathbf{2}$ was thus assigned as shown.

The tentative phytotoxic activity of $\mathbf{1}$ and $\mathbf{2}$ was evaluated by determining their effect on the radicle elongation of germinating lettuce. At 200 ppm, $\mathbf{2}$ inhibited radicle length of lettuce up to $30 \%$ as compared to the distilled $\mathrm{H}_{2} \mathrm{O}$ control, while 1 caused $11 \%$ growth inhibition. These results suggest that compounds $\mathbf{1}$ and $\mathbf{2}$ play an allel opathic role in M. tanarius.

\section{Experimental Section}

General Experimental Procedures. IR spectra were recorded on a Perkin-E Imer $983 \mathrm{G}$ spectrophotometer. ${ }^{1} \mathrm{H}$ and ${ }^{13} \mathrm{C}$ NMR spectra were run on a Varian Unity Plus 400 spectrometer. EIMS and specific rotations were taken on a J EOL J MS-HX 300 mass spectrometer and a J ASCO DIP-1000 digital polarimeter, respectively. Extracts were chromatographed on silica gel (MERK 70-230, 230-400 mesh, ASTM).

Plant Material. The fallen leaves of $M$. tanarius were collected from the campus of National Taiwan University, Taiwan, in 1998. A voucher specimen (voucher no. 01566) has been deposited at the Herbarium of the Department of Mathematics and Science Education, Taipei Municipal Teachers College, Taipei, Taiwan.

Extraction and Isolation. The dried fallen leaves of $M$. tanarius were ground to powder, and $4 \mathrm{~kg}$ of the powder was extracted with $\mathrm{MeOH}(100 \mathrm{~L})$ at room temperature $(7$ days $x$ 2). The extract was evaporated in vacuo to yield a residue, which was suspended in $\mathrm{H}_{2} \mathrm{O}(1 \mathrm{~L})$, and this was partitioned with ethyl acetate $(1 \mathrm{~L} \times 3)$. The combined ethyl acetate layer afforded a black syrup ( $250 \mathrm{~g})$ that was subsequently chromatographed over silica gel using a hexane/EtOAc gradient solvent system. Crude compounds $\mathbf{1}, \mathbf{2}$, and $\mathbf{3}$ were all eluted with $30 \%$ EtOAc in hexane. Further purification by HPLC (Merck LichroCART 250-10 Cat. 1.50179 Lichrosorb Si 60 (7 $\mu \mathrm{m})$ ) gave 1 (30 mg), 2 (7 mg), and 3 (500 mg) using 30\% EtOAc/hexane.

Bioassays. A 200 ppm solution of each pure isolated compound in $\mathrm{MeOH}$ was prepared for bioassay. The solutions were spread onto the silica TLC sheets $(1 \mathrm{~cm} \times 5 \mathrm{~cm})$, allowing the $\mathrm{MeOH}$ to evaporate completely in a laminar flow hood. Prior to bioassay, the TLC sheets were placed in Petri dishes and moistened with distilled $\mathrm{H}_{2} \mathrm{O}$, then surrounded by wet sponge without contact. Lettuce (Lactuca sativa L. var. Iongifolia Lam.) seeds were imbibed in distilled $\mathrm{H}_{2} \mathrm{O}$ for $2 \mathrm{~h}$, then placed on the TLC sheets containing the pure compound or on untreated control sheets. The Petri dishes were sealed with M Parafilm (American National Can), then placed in an incubator at $25^{\circ} \mathrm{C}$ for $48 \mathrm{~h}$ in dark.

Tanariflavanone A (1): greenish oil; $[\alpha]^{24.6}+26.8^{\circ}$ (c 0.6 , $\left.\mathrm{CHCl}_{3}\right) ; \mathrm{UV}(\mathrm{MeOH}) \lambda_{\max }(\log \epsilon) 231 \mathrm{sh}$ (4.34), 293 (4.29) nm; IR (KBr) $v_{\max } 3422,1647,1610,1500,1457 \mathrm{~cm}^{-1}$; ${ }^{1} \mathrm{H}$ NMR $(400$ $\left.\mathrm{MHz}_{2} \mathrm{CDCl}_{3}\right) \delta 1.31,1.35,1.57,1.64,1.74$ (each 3H, s), 2.06 (4H, m, H-5",-6"), $2.64\left(1 \mathrm{H}, \mathrm{dd}, \mathrm{J}=16.8,5.2 \mathrm{~Hz}, \mathrm{H}_{\mathrm{a}}-\mathrm{I}^{\prime \prime \prime}\right), 2.73$ $\left(1 \mathrm{H}, \mathrm{dd}, \mathrm{J}=17.2,2.8 \mathrm{~Hz}, \mathrm{H}_{\mathrm{eq}}-3\right), 2.87(1 \mathrm{H}, \mathrm{dd}, \mathrm{J}=16.8,5.2$ $\left.\mathrm{Hz}, \mathrm{H}_{\mathrm{b}}-^{\prime \prime \prime}\right), 3.12\left(1 \mathrm{H}, \mathrm{dd}, \mathrm{J}=17.2,12.8 \mathrm{~Hz}, \mathrm{H}_{\mathrm{ax}}-3\right), 3.42(2 \mathrm{H}$, br d, J = $\left.6.8 \mathrm{~Hz}, \mathrm{H}-\mathrm{I}^{\prime \prime}\right), 3.83\left(\mathrm{H}, \mathrm{t}, \mathrm{J}=5.2 \mathrm{~Hz}, \mathrm{H}-2^{\prime \prime \prime}\right), 5.02$ $\left(1 \mathrm{H}, \mathrm{br}, \mathrm{t},=6.8 \mathrm{~Hz}, \mathrm{H}-7^{\prime \prime}\right), 5.17\left(1 \mathrm{H}, \mathrm{br} \mathrm{t}, \mathrm{J}=6.6 \mathrm{~Hz}, \mathrm{H}-2^{\prime \prime}\right)$, $5.48(1 \mathrm{H}, \mathrm{dd}, \mathrm{J}=12.8,2.8 \mathrm{~Hz}, \mathrm{H}-2), 5.94(1 \mathrm{H}, \mathrm{s}, \mathrm{H}-8), 6.80$, 6.96 (each $1 \mathrm{H}, \mathrm{d}, \mathrm{J}=8.4 \mathrm{~Hz}, \mathrm{H}-5^{\prime},-6^{\prime}$ ), 5.56, 5.89, 12.35 (each $1 \mathrm{H}, \mathrm{s}, \mathrm{OH}) ;{ }^{13} \mathrm{C}$ NMR $\left(100 \mathrm{MHz} \mathrm{CDCl}_{3}\right) \delta 196.4(\mathrm{~s}, \mathrm{C}-4), 161.9$ (s, C-9), 161.5 (s, C-7), 160.8 (s, C-5), 144.8 (s, C-4'), 142.5 (s, C-3'), 138.9 (s, C-3"), 132.2 (s, C-8'), 128.3 (s, C-1'), 126.4 (s, C-2'), 123.7 (d, C-7"), 121.3 (d, C-2"), 118.9 (d, C-6'), 112.9 (d, C-5'), 102.7 (s, C-6), 99.9 (s, C-10), 96.2 (d, C-8), 78.6 (s, C-3"') 76.9 (d, C-2), 68.8 (d, C-2'"), 42.4 (t, C-3), 39.6 (t, C-5"), 26.3 $\left(\mathrm{t}, \mathrm{C}-6^{\prime \prime}\right), 25.7\left(\mathrm{q}, \mathrm{C}-10^{\prime \prime}\right), 25.4\left(\mathrm{t}, \mathrm{C}-1^{\prime \prime}\right), 25.0\left(\mathrm{q}, \mathrm{C}-4^{\prime \prime \prime}\right), 24.9(\mathrm{t}$, C-1"'), 22.0 (q, C-5'"'), 17.7 (q, C-9"'), 16.2 (q, C-4"); EIMS m/z $508\left[^{M}\right]^{+}(33), 490(10), 423$ (50), 384 (29), 237 (100); HREIMS $\mathrm{m} / \mathrm{z} 508.2446$ (calcd for $\mathrm{C}_{30} \mathrm{H}_{36} \mathrm{O}_{7}, 508.2451$ ).

Tanariflavanone B (2): brownish oil; $[\alpha]^{24.6}{ }_{\mathrm{D}}+28.2^{\circ}(\mathrm{c} 0.5$, $\left.\mathrm{CHCl}_{3}\right) ;$ I R $v_{\max } 3374,1641,1606,1449,1156 \mathrm{~cm}^{-1}$; UV (MeOH) $\lambda_{\max }(\log \epsilon) 227$ (4.83), 274 (4.47), 285 (4.49) nm; ${ }^{1} \mathrm{H}$ NMR (400 $\left.\mathrm{MHz} \mathrm{CDCl}_{3}\right) \delta 1.41,1.55,1.65,1.74,1.80$ (each 3H, s), 2.08 $\left(4 \mathrm{H}, \mathrm{m}, \mathrm{H}-5^{\prime \prime},-6^{\prime \prime}\right), 2.73\left(1 \mathrm{H}, \mathrm{dd}, \mathrm{J}=17.2,2.8 \mathrm{~Hz}, \mathrm{H}_{\mathrm{eq}}-3\right), 3.11$ $\left(1 \mathrm{H}, \mathrm{dd}, \mathrm{J}=17.2,13.2 \mathrm{~Hz}, \mathrm{H}_{\mathrm{ax}}-3\right), 3.32(2 \mathrm{H}, \mathrm{br} \mathrm{d}, \mathrm{J}=5.8 \mathrm{~Hz}$, $\left.\mathrm{H}-\mathrm{I}^{\prime \prime \prime}\right), 5.07\left(\mathrm{H}, \mathrm{br} \mathrm{t}, \mathrm{J}=5.7, \mathrm{H}-\mathrm{7}^{\prime \prime}\right), 5.23(\mathrm{H}, \mathrm{br} \mathrm{t}, \mathrm{J}=5.8 \mathrm{~Hz}$, $\left.\mathrm{H}-2^{\prime \prime \prime}\right), 5.48(1 \mathrm{H}, \mathrm{dd}, \mathrm{J}=13.2,2.8 \mathrm{~Hz}, \mathrm{H}-2), 5.65(1 \mathrm{H}, \mathrm{d}, \mathrm{J}=$ $\left.10.0 \mathrm{~Hz}, \mathrm{H}-2^{\prime \prime}\right), 5.97(1 \mathrm{H}, \mathrm{s}, \mathrm{H}-8), 6.58(1 \mathrm{H}, \mathrm{d}, \mathrm{J}=10.0 \mathrm{~Hz}$, $\left.\mathrm{H}-\mathrm{I}^{\prime \prime}\right), 6.81\left(1 \mathrm{H}, \mathrm{d}, \mathrm{J}=8.4 \mathrm{~Hz}, \mathrm{H}-5^{\prime}\right), 6.89(1 \mathrm{H}, \mathrm{d}, \mathrm{J}=8.4 \mathrm{~Hz}$, $\left.\mathrm{H}-6^{\prime}\right) ; 5.66,6.32,12.38$ (each $\left.1 \mathrm{H}, \mathrm{s}, \mathrm{OH}\right) ;{ }^{13} \mathrm{C}$ NMR $(100 \mathrm{MHz}$ $\left.\mathrm{CDCl}_{3}\right) \delta 196.3$ (s, C-4), 163.7 (s, C-7), 161.3 (s, C-9), 161.0 (s, C-5), 145.1 (s, C-4'), 139.7 (s, C-3'), 135.3 (s, C-3'”'), 132.1 (s, C-8'), 130.9 (d, C-2"'), 124.8 (s, C-1'), 123.7 (d, C-7'), 121.5 (d, C-2'") 118.9 (d, C-1") 118.9 (d, C-6'), 118.8 (s, C-2'), 114.5 (d, C-5'), 107.2 (s, C-6), 102.9 (s, C-10), 95.5 (d, C-8), 79.0 (s, C-3"), 76.0 (d, C-2), 42.5 (t, C-3), 40.7 (t, C-5"), 26.1 (q, C-4"), 25.8 (q, C-4"'), 25.6 (q, C-10"), 22.7 (t, C-6"), 21.1 (t, C-1"'), 17.8 (q, C-5"'), 17.6 (q, C-9"); EIMS m/z 490 [M] ${ }^{+}$(17), 407 (100), 219 (26); HREIMS m/z 490.2374 (calcd for $\mathrm{C}_{30} \mathrm{H}_{34} \mathrm{O}_{6}, 490.2346$ ).

Acknowledgment. This work was supported by grants (NSC-88-2311-B-001; NSC-89-2311-B-001-026) to C.H.C. awarded by the National Science Council of Republic of China.

\section{References and Notes}

(1) Webster, G. Ann. Missouri Bot. Garden 1994, 81, 33-144.

(2) Hui, W. H.; Ng, K. K.; Fukamiya, N.; Koreeda, M.; Nakanishi, K. Phytochemistry 1971, 10, 1617-1620.

(3) Hui, W. H.; Li, M. M.; N g, K. K. Phytochemistry 1975, 14, 816-817.

(4) Lin, J . H.; Nonaka, G. I.; Nishioka, I. Chem. Pharm. Bull. 1990, 38, $1218-1223$.

(5) Thoison, O.; Hnawia, E.; Guéritte-Voegelein, F.; Sévenet, T. Phytochemistry 1992, 31, 1439-1442.

(6) Hnawia, E.; Thoison, O.; Guéritte-Voegelein, F.; Bourret, D.; Sévenet, T. Phytochemistry 1990, 29, 2367-2368.

(7) Schütz, B. A.; Wright, A. D.; Rali, T.; Sticher, O. Phytochemistry 1995 40, 1273-1277.

(8) Sultana, S.; Ilyas, M. Phytochemistry 1986, 25, 953-954.

(9) Beutler, J. A.; Shoemaker, R. H.; J ohnson, T.; Boyd, M. R. J . Nat Prod. 1998, 61, 1509-1512.

(10) Hsieh, C. F. Flora of Taiwan; Epoch Publish Co., Ltd.: Taipei, 1977; Vol. III, p 479

(11) Yakushijin, K.; Shibayama, K.; Murata, H.; Furukawa, H. Heterocycles 1980, 14, 397-402.

(12) Gaffield, W. Tetrahedron 1970, 26, 4093-4108

(13) Huang, L.; Fullas, F.; McGivney, R. J .; Brown, D. M.; Wani, M. C. Wall, M. E. J. Nat. Prod. 1996, 59, 290-292.

NP0100338 